IN PRACTICE

\title{
Methods employed by genitourinary medicine clinics in the United Kingdom to diagnose bacterial vaginosis
}

\author{
F E A Keane, R Maw, C Pritchard, C A Ison
}

Sex Transm Infect 2005;81:155-157. doi: 10.1136/sti.2004.009902

See end of article for authors' affiliations

......................

Correspondence to:

Dr Frances Keane,

Department of

Genitourinary Medicine

Royal Cornwall Hospital,

Treliske, Truro TR1 3L,

UK; keanefr@rcht.

cornwall.nhs,uk

Accepted for publication 3 June 2004

\begin{abstract}
Objective: To determine the methods used by genitourinary medicine (GUM) clinics in the United Kingdom for the diagnosis of bacterial vaginosis (BV).

Methods: A questionnaire survey of UK GUM clinics was conducted.

Results: 148/221 (67\%) clinics returned a questionnaire. 96/148 (64.9\%) clinics reported using Amsel's criteria to diagnose BV but only $29(30.5 \%)$ of these used all four of the composite criteria. 139/148 $(93.9 \%)$ clinics used the appearance of a Gram stained vaginal smear as an aid in BV diagnosis, although a variety of scoring methods was employed. In the majority of clinics, 92/148 (62.2\%), one staff discipline provided the microscopy service, in 50 (33.8\%) clinics two staff disciplines provided microscopy services. The bulk of microscopy services within UK GUM clinics is provided by nurses.

Conclusions: Most UK GUM clinics utilise the appearance of a Gram stained vaginal smear for the diagnosis of BV although there is little consensus at present about the type of scoring method employed. Adaptation of a uniform scoring method would have enormous benefits, including consistency and reproducibility of results and the development of quality assurance schemes for BV diagnosis on a national basis. There are important issues to be addressed regarding the initial training and ongoing support for nurses providing microscopy services within UK GUM clinics.
\end{abstract}

B acterial vaginosis (BV) is characterised by an alteration in the vaginal ecology where the normal Lactobacillus dominated flora is replaced by a mixed bacterial flora that includes Gardnerella vaginalis, Mycoplasma hominis, and anaerobes such as Mobiluncus spp and Prevotella spp. Although the condition was first described (at that time as Haemophilus vaginalis vaginitis) in 1955 by Gardener and Dukes ${ }^{1}$ it was not until 1983 that Amsel et al ${ }^{2}$ proposed standardised diagnostic criteria. This method, which has remained the gold standard for the diagnosis of BV until recent times, proposed that BV was diagnosed on the presence of three out of four composite criteria - that is, a characteristic thin, homogeneous vaginal discharge, vaginal $\mathrm{pH}$ above 4.5 , a positive "whiff" test, and the presence of clue cells on dark ground microscopy of a saline wet mount preparation. In the same year, Spiegel et al, ${ }^{3}$ of whom Amsel was one, published their work on the use of the appearance of a Gram stained vaginal smear for the diagnosis of BV. They assigned a simple scoring system based on the presence of Lactobacillus spp, Gardnerella spp, and other morphotypes. A direct comparison of the two methods by Eschenbach et al found varying sensitivities (43\% to $97 \%$ ) and specificities (53\% to $99 \%$ ) of each of the four criteria when compared to Spiegel's Gram stain criteria. Since then, several other scoring systems, including Nugent's, ${ }^{5}$ a modified Spiegel's (Hay-Ison), ${ }^{6}$ and a modified Ison-Hay ${ }^{7}$ method have been proposed and validated.

Genitourinary medicine (GUM) clinics in the United Kingdom routinely perform in-house diagnosis of BV. Until now, their usage of the available diagnostic tools has not been studied. A recent pilot external quality assurance scheme for the diagnosis of BV on the appearance of Gram stained vaginal smears revealed little consistency of scoring system preference between the five participating centres (F Keane, personal communication). The following questionnaire survey was designed to investigate current diagnostic practices for BV within UK GUM clinics.

\section{METHODS}

A questionnaire (designed by FK) was distributed by the British Co-operative Clinical Group to all lead clinicians in UK GUM clinics. They were surveyed about the use of Amsel's criteria, examination of a Gram or acridine orange stained vaginal smear, or other method to diagnose BV in their routine practice. Clinics using the appearance of Gram stained vaginal smears were asked to disclose their scoring system. Information was also gathered on the staff disciplines providing routine microscopy services within each clinic and the use of microbiology laboratories to provide a diagnostic service for BV to GUM clinics. Data were analysed using the SPSS statistical package.

\section{RESULTS}

Replies were received from clinicians responsible for 148/221 $(67 \%)$ clinics.

\section{Diagnostic methods for BV}

A total of 96/148 (64.9\%) clinics reported using Amsel's composite criteria to diagnose BV. One of these did not detail how many of the criteria it routinely employed. Of the remaining 95 clinics, $10(10.5 \%)$ reported using only one of the criteria, $27(28.4 \%)$ two, $29(30.5 \%)$ three, and only 29 $(30.5 \%)$ reported using all four of the criteria necessary to make a diagnosis of $\mathrm{BV}$ by this method. The use of the individual criteria is detailed in table 1 . Of the $25(16.9 \%)$ replying no to the use of Amsel's criteria and the 27 (18.2\%) who left the reply blank, a substantial proportion reported the use of at least some of the composite criteria (table 1); $139 / 148(93.9 \%)$ clinics reported using examination of a Gram stained vaginal smear for the diagnosis of BV; 44 $(31.7 \%)$ of these did not report any scoring method. Of the

Abbreviations: BV, bacterial vaginosis; GUM, genitourinary medicine; MLSOs, medical laboratory scientific officers 


\begin{tabular}{llll|}
\hline \multicolumn{2}{l}{ Table 1 Use of Amsel's criteria } & & \\
\hline $\begin{array}{l}\text { Component of Amsel's } \\
\text { criteria }\end{array}$ & $\begin{array}{l}\text { Yes } \\
(\mathbf{n}=96)(64.9 \%)\end{array}$ & $\begin{array}{l}\text { No } \\
(\mathbf{n}=25)(16.9 \%)\end{array}$ & $\begin{array}{l}\text { Blank reply } \\
(\mathbf{n}=\mathbf{2 7})(\mathbf{1 8 . 2} \%)\end{array}$ \\
\hline Discharge & $91(95.8)$ & $11(44.0)$ & $26(96.3)$ \\
pH & $58(61.1)$ & $2(8.0)$ & $13(48.1)$ \\
"Whiff test" & $37(38.9)$ & $4(16.0)$ & $5(18.5)$ \\
Clue cells on "wet" & $81(85.3)$ & $9(36.0)$ & $24(88.9)$ \\
preparation & & & \\
\hline
\end{tabular}

remaining 95 clinics, 94 employed one scoring system alone, but one clinic reported five different methods. Of the 99 scoring methods employed-24 were Nugent's scoring system, eight Spiegel's, 33 Hay/Ison method, and 34 "some other" scoring method.

Only five $(3.3 \%)$ clinics used the appearance of an acridine stained vaginal smear for the diagnosis of BV. Ten clinics reported "other" diagnostic methods," consisting of various combinations of the above and the use of anaerobic cultures in two cases. Samples were sent to microbiology laboratories by $36 / 148(24 \%)$ clinics to aid in the diagnosis of BV. Of these, 14 sent air dried and nine Gram stained vaginal smears respectively; 31 sent charcoal swabs for culture of Gardenerella vaginalis.

\section{Staff disciplines performing microscopy}

The microscopy service was provided by one staff discipline in $92 / 148(62.2 \%)$ clinics; in $49(53.3 \%)$ of these clinics by nurses, $27(29.3 \%)$ by medical laboratory scientific officers (MLSOs), 15 (16.3\%) by doctors, and in one by some "other" unspecified staff member. In 50 (33.8\%) clinics, microscopy services were provided by two different staff disciplines: in 34 $(68 \%)$ of these clinics by doctors and nurses, in six $(12 \%)$ by doctors and MLSOs, in six (12\%) by nurses and MLSOs, and in four (by doctors and "other"). In five clinics there was a

\section{Key messages}

- This survey, which examines current diagnostic practice for BV in UK GUM clinics, reveals the following:

- the use of Amsel's criteria is largely unsatisfactory as most clinics do not employ all of the criteria-an essential prerequisite of this method

- most UK clinics use the appearance of a Gram stained vaginal smear to aid in the diagnosis of BV, but there is little consensus about the scoring methods they employ

- the bulk of microscopy services within UK GUM clinics are provided by nurses

- The authors recommend the universal adoption of the Ison-Hay scoring system to

- improve diagnostic consistency across clinics

- assist in the development /implementation of both internal and external quality assurance schemes for the diagnosis of BV

- strengthen the body of BV research in the UK as studies

- There are important issues to be addressed regarding the initial training and on-going support for nurses providing microscopy services within UK GUM clinics. multidisciplinary provision of microscopy services and one clinic had no "in-house" laboratory service.

\section{DISCUSSION}

This is the first survey to examine routine "in-house" diagnostic methods for BV within UK GUM clinics. The data regarding the use of Amsel's criteria are difficult to interpret: less than a third of those using this method actually employed all of the composite criteria to do so, thus, by definition, invalidating the method. This would suggest that the use of Amsel's criteria is not the method of choice for diagnosing $\mathrm{BV}$ in routine clinical practice. Interestingly, the overwhelming majority of survey respondents were using the appearance of a Gram stained vaginal smear to aid their diagnosis of BV. However, although there was an unwritten consensus that the Gram stain was the most useful method for the diagnosis of $\mathrm{BV}$, there was poor agreement about the most suitable scoring system. An important caveat to mention is that although the appearance of the vaginal Gram stain should be used to interpret a patient's vaginal flora; the result needs to be viewed in a clinical context to establish whether a patient requires treatment. It is well accepted that $G$ vaginalis can be isolated from women both with and without $\mathrm{BV}^{48}$ and thus sending cultures for $G$ vaginalis is unhelpful in trying to establish a diagnosis of $\mathrm{BV}$.

The adoption of a single scoring system for the diagnosis of BV on Gram stained vaginal smears by all GUM clinics in the United Kingdom would be extremely advantageous. Firstly, from the point of consistency; there would be increased confidence that a Gram stain vaginal smear taken in any UK clinic would be interpreted in a similar manner. Secondly, adoption of the one system would aid enormously in the development/implementation of both internal and external quality assurance schemes for the diagnosis of BV in UK GUM clinics. Furthermore, use of a single scoring system would strengthen the body of BV research in the United Kingdom as studies would, by definition, become more directly comparable than they are at present. Most BV researchers now favour the Gram stain method over Amsel's criteria; slides may be stored and both Gram stained and read in batches. The method offers convenience and an opportunity for confirmation of findings that is lost when Amsel's method is employed.

Ison and Hay have validated their modified simple grading system $^{7}$ against both Amsel's criteria ${ }^{2}$ and Nugent's method in an international comparison ${ }^{9}$ and have found excellent correlations. The Ison-Hay method can be easily incorporated into routine clinic practice, unlike Nugent's scoring system, which requires the counting of different types of bacteria, making it too time consuming for use in a busy clinical setting. The Ison-Hay method is recommended by the Bacterial Special Interest Group (BSIG) as the method of choice for the diagnosis of BV in GUM clinics. It has also been recommended for screening for $\mathrm{BV}$ in the guidelines for screening for sexually transmitted infections (Clinical Effectiveness Group/BSIG of BASHH, in preparation). Uniform adoption of the Ison-Hay method across UK GUM 
clinics could achieve the desired consistency of BV diagnosis in areas of both clinical and research practice and assist in the development of robust quality assurance schemes for BV diagnosis in GUM clinic laboratories.

The survey also revealed that GUM nurses provide the bulk of microscopy services with UK GUM clinics. This raises important issues regarding initial training, on-going support and clinical governance for the delivery of microscopy services within GUM services.

\section{ACKNOWLEDGEMENTS}

The authors would like to thank the Bacterial Special Interest Group of the British Association for Sexual Health and HIV and the British Co-operative Clinical Group for their support of this project and are indebted to all the clinics that completed the survey questionnaires.

\section{CONTRIBUTORS}

FK designed the questionnaire, performed the initial statistical analysis, and wrote the manuscript; RM contributed to the questionnaire design, distributed the questionnaire, collated the responses, and commented on the manuscript; CP carried our more detailed statistical analysis; CI contributed to the questionnaire design and the discussion section of the manuscript.

\section{Authors' affiliations}

F E A Keane, Department of Genito-urinary Medicine, Royal Cornwall Hospital, Truro, UK

R Maw, Department of Genito-urinary Medicine, Royal Victoria Hospital, Belfast, UK
C Pritchard, Research and Development Support Unit, Royal Cornwall Hospital, Truro, UK

C A Ison, Department of Infectious Diseases and Microbiology, Imperial College London, St Mary's Campus, London, UK

Conflict of interest: None.

\section{REFERENCES}

1 Gardner HL, Dukes CD. Haemophilus vaginalis vaginitis. A newly defined specific infection previously classified "non-specific" vaginitis. Am J Obstet Gynecol 1955;69:962-76.

2 Amsel R, Totten PA, Spiegel CA, et al. Nonspecific vaginitis: diagnostic criteria and microbial and epidemiological associations. Am J Med 1983;74:14-22.

3 Spiegel CA, Amsel R, Holmes KK. Diagnosis of bacterial vaginosis by direct Gram stain of vaginal fluid. J Clin Microbiol 1983;18:170-7.

4 Eschenbach DA, Hillier S, Critchlow C, et al. Diagnosis and clinical manifestations of bacterial vaginosis. Am J Obstet Gynecol 1988;158:819-28.

5 Nugent RP, Krohn MA, Hillier SL. Reliability of diagnosing bacterial vaginosis is improved by a standardised method of Gram-stain interpretation. J Clin Microbiol 1991;29:297-301.

6 Hay PE, Lamont RF, Taylor-Robinson D, et al. Abnormal bacterial colonisation of the genital tract and subsequent preterm delivery and late miscarriage. BMJ 1994;308:295-8.

7 Ison CA, Hay PE. Validation of a simplified grading of Gram-stained vaginal smears for use in genitourinary medicine clinics. Sex Transm Infect 2002; 78:413-5.

8 Totten PA, Amsel R, Hale J, et al. Selective differential human blood bilayer media for isolation of Gardnerella (Haemophilus) vaginalis. J Clin Microbiol 1982;15:141-7.

9 Forsum U, Jakobsson T, Larsson PG, et al. An international study of the interobserver variation between interpretations of vaginal smear criteria of bacterial vaginosis. APMIS 2002;110:811-8. 